\title{
A New Auto Exposure System to Detect High Dynamic Range Conditions Using CMOS Technology
}

\author{
Quoc Kien Vuong, Se-Hwan Yun and Suki Kim \\ Korea University, Seoul \\ Republic of Korea
}

\section{Introduction}

Recently, Image Signal Processing (ISP) has become an interesting research field, along with the development and emergence of various image capturing systems. These image systems include digital still cameras, surveillance systems, webcams, camcorders, etc... ISP is any form of signal processing for which the input is an image, such as photographs or frames of video; the output of image processing can be either an image or a set of characteristics or parameters related to the image. Most image-processing techniques involve treating the image as a two-dimensional signal and applying standard signal-processing techniques to it. ISP helps visually optimize raw output images captured with image sensors located in image systems.

For most of such devices, auto exposure (AE) has become one major function which automatically adjust the amount of incident light on the image sensor so as to utilize its full dynamic range, or for proper exposure. To control the amount of incident light, cameras adjust the aperture, shutter speeed, or both. If the expsoure time is not long enough, output images will appear darker than actual scenes, which is called under-exposure. On the other hand, if the exposure time is too much, output images will appear much brighter than actual scene, which is called over-exposure. Both cases result in a loss of details and image would pocess a bad quality. Only at an appropriate exposure can a camera provide good pictures with the most details.

Many AE algorithms have been developed (Liang et al., 2007), (Shimizu et al., 1992), (Murakami \& Honda, 1996) and (Lee et al., 2001) to deal with high-contrast lighting conditions. Some of them employ fuzzy method while others use various ways of segmentation. However, most of these algorithms have some drawbacks on either their accuracy or on the complexity, or both while estimating lighting conditions.

According to the research (Liang et al., 2007), it is difficult to discriminate back-lit conditions from front-lit conditions using histogram methods (Shimizu et al., 1992) and (Murakami \& Honda, 1996). Further simulations in this paper shows that the tables and criteria used to estimate lighting conditions are confusing and not consistent. These methods tend to address only excessive back-lighting and front-lighting conditions as well as how to distinguish between these two conditions.

Source: Convergence and Hybrid Information Technologies, Book edited by: Marius Crisan, ISBN 978-953-307-068-1, pp. 426, March 2010, INTECH, Croatia, downloaded from SCIYO.COM 


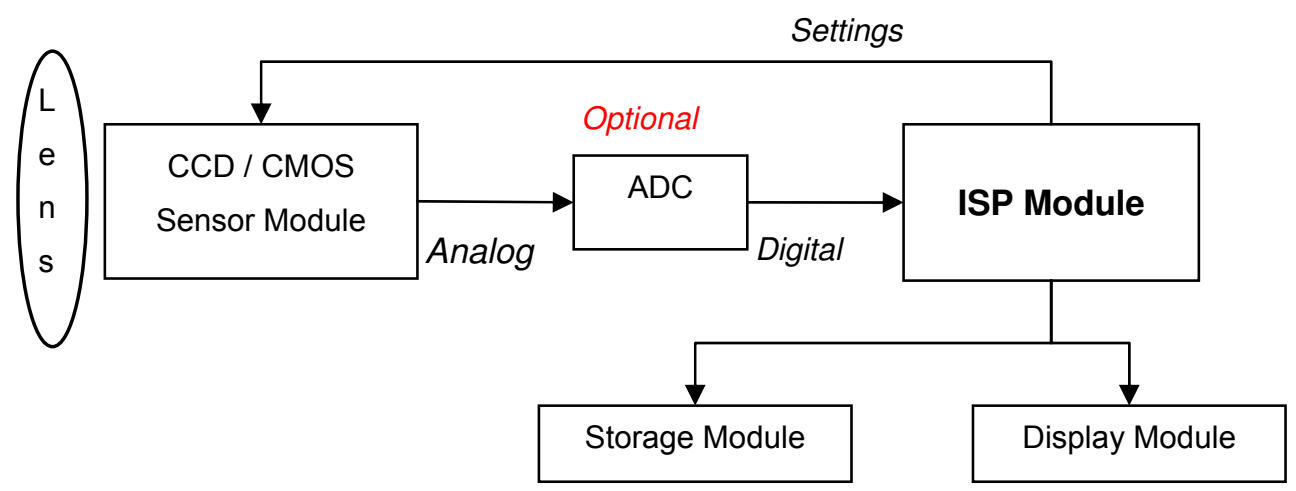

Fig. 1. Simplified block diagram of an image capturing system

Other algorithms such as (Murakami and Honda, 1996) and (Lee et al., 2001) used fixedwindow segmentation methods to estimate the brightness and lighting conditions. The main drawback of these algorithms is the in-flexibility. Most of these algorithms, including (Liang et al., 2007) assume that there is a main object in each image; therefore, they can not work well with images that have no main objects, only normal sceneries, or images in which a main object is not located at the centre. Furthermore, the gain coefficients for each region in a picture are different, hence color and brightness distortion may occur.

In (Kao et al., 2006), multiple exposure methods were presented to improve the dynamic range of output pictures. Simulation results showed that its algorithm might easily lead to color inconsistency and bad chromatic transitions.

This paper introduces a new approach to control AE which can be used to determine the degree of contrast lighting employing a simple and quick method which is presented in Section 3. Section 4 describes how to decide if the condition is normal lit, excessive back lit or just a condition with a high dynamic range. Then the algorithm uses a simple multiple exposure mechanism to improve the dynamic range of the output image so that more details can be revealed. In Section 5, simulation results are presented. Finally, conclusions are given in Section 6.

\section{AE algorithm for lighting-condition detection}

\subsection{Lighting condtion detecting}

Lighting conditions can be generally classified as normal-lit, excessive back-lit or high contrast. A back lighting condition is a scene in which light sources are located behind the whole scenery or main objects. In this case, the brightness of the background is much higher than that of the main object. A high contrast lighting condition is a scene that consists of many regions of very different brightness levels. Front lighting conditions can also be considered as high contrast lighting. These are the conditions in which light sources are located in front of and somehow close to the main object and therefore, the brightness of that main object is much higher than that of the background.

Usually, it is not very difficult at all to capture images of normal lit or normal illuminated scenes. However, in the cases of excessive back-lit and high contrast lighting conditions, output images may lose a significant amount of details. A picture taken in such a condition may contain regions that are much darker or brighter than the actual ambient scene. If the 
exposure value is set such that dark objects and regions look bright enough to see, then other bright objects and regions will be too bright or over-exposed. On the contrary, if the exposure value is set such that bright objects and areas become adequately bright enough to human eyes, then other objects and areas will be too dark or under-exposed to distinguish each separate detail. Estimating litghting conditions accurately can help a camera device decide how to compensate its exposure value for better output pictures.

To determine the degree of lighting conditions, the proposed method uses the relationship between the mean value and median value of an image.

The mean value is simply the average component value of all elements in an array, or particularly of all pixels in an image. A component can be a color component $(R, G$, or $B)$ or the brightness level.

The median value is the value of the middle element in a sorted array. This array is an array of brightness levels of all pixels in an image. Note that since the element at the middle is taken into account, the array can be sorted either ascendingly or descendingly without affecting the value of the middle element. Fig. 2 illustrates the difference between these two values.

Original array
\begin{tabular}{|l|l|l|l|l|l|l|l|l|}
\hline 158 & 250 & 85 & 203 & 70 & 89 & 110 & 105 & 120 \\
\hline
\end{tabular}

Sorted array

\begin{tabular}{|l|l|l|l|l|l|l|l|l|}
\hline 70 & 85 & 89 & 105 & 110 & 120 & 158 & 203 & $250 \quad$ Median value: 110 \\
\hline
\end{tabular}

Fig. 2. Mean and median values of an array

According to Fig. 2, although the average value is somewhere in the middle of the range, the median value is much smaller than the mean value. This is because the number of small value elements outweights that of large value ones. For a sorted large-size array, if the values of all elements increase or decrease steadily, the difference between the mean and the median values is not significant. However, if the values of all items increase or decrease abruptly somewhere within the array, then the middle item may have a very large or very small value, depending on the outweighing number of large-value or small-value elements. This leads to a significant difference between the mean and the median values.

The idea of estimating the relationship between the mean and median values of an array can be applied to lighting condition detection. Since the total number of pixels in an image is very large, that idea will be even more accurate and applicable. In the case of normal lighting conditions, the brightness level of all pixels follows a steady distribution throughout the whole color and brightness ranges of each image. Therefore, the mean value just differs a little from the median value. On the contrary, in the cases of high contrast lighting and back lighting conditions, for under- or appropriate exposure value, the median value of the brightness levels tends to reside in the small-value section and hence, it differs much from the average value of the whole array of all pixels.

Fig. 3 illustrates the use of the relationship between these two values in detecting illuminating conditions. Note that $B l_{\text {mean }}$ and $B l_{\text {med }}$ denote the mean and the median value of the brightness level, respectively, $D_{L}$ denotes the difference between the two values, and $D_{\text {thres }}$ denotes the threshold value. 


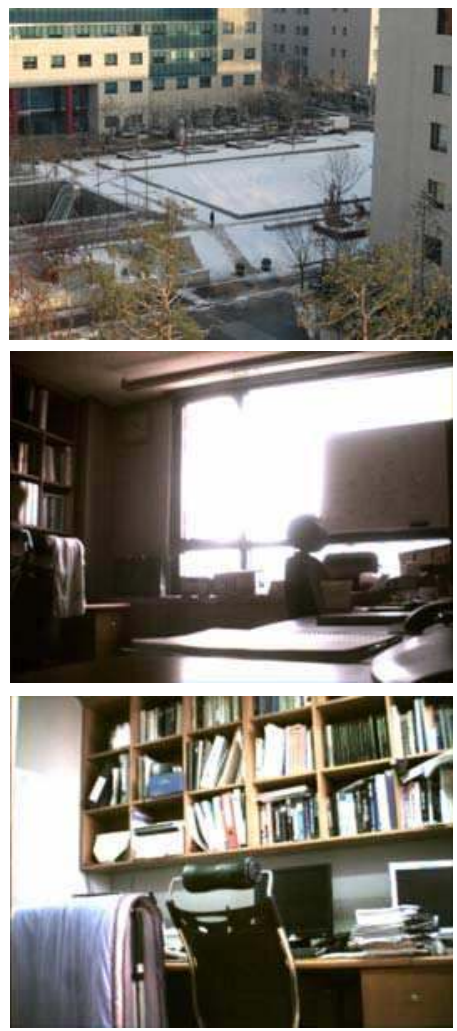

(a) Normal-lighting

$$
\begin{aligned}
& B I_{\text {mean }}=112 \\
& B I_{\text {med }}=103 \\
& D_{L}=9<D_{\text {thres }}
\end{aligned}
$$

(b) Back-lighting

$B I_{\text {mean }}=118$

$B I_{\text {med }}=79$

$D_{L}=39>D_{\text {thres }}$

(c) High Contrast Lighting

$$
\begin{aligned}
& B I_{\text {mean }}=120 \\
& B I_{\text {med }}=100 \\
& D_{L}=20 \geq D_{\text {thres }}
\end{aligned}
$$

Fig. 3. $B l_{\text {mean }}, B l_{\text {med }}$ and $D_{L}$ in different lighting conditions

The next issue is to decide the value of brightness level of an image. Unlike most high end camera systems, low end camera platforms employ CMOS image sensors that produce output images in the RGB form. Most conventional systems perform the conversion from RGB to another color space such as $\mathrm{YCbCr}$ in order to reveal the luminance value $\mathrm{Y}$. However, since the green component $(\mathrm{G})$ contributes the most to the brightness of an image, $\mathrm{G}$ can be used directly as the brightness level without introducing much difference from $\mathrm{Y}$. This can help reduce the complexity and processing time of the overall architecture. Experimental results of (Liang et al., 2007) demonstrate the similarity between $Y$ and G. Referring back to Fig. 3, all brightness values $\left(B l_{\text {mean }}, B l_{\text {med }}\right)$ are exactly values of $Y$ (luminance) component of each image. The following table provides corresponding brightness values in term of $\mathrm{G}$ component for images in Fig. 3.

\begin{tabular}{|c|c|c|c|c|c|c|}
\hline \multirow{2}{*}{ Image } & \multicolumn{2}{|c|}{$B \boldsymbol{l}_{\text {mean }}$} & \multicolumn{2}{c|}{$\boldsymbol{B} \boldsymbol{l}_{\text {med }}$} & \multicolumn{2}{c|}{$\boldsymbol{D}_{\mathbf{L}}$} \\
\cline { 2 - 7 } & $\mathbf{G}$ & $\mathbf{Y}$ & $\mathbf{G}$ & $\mathbf{Y}$ & $\mathbf{G}$ & $\mathbf{Y}$ \\
\hline $\mathbf{( a )}$ & 111 & 112 & 103 & 103 & 8 & 9 \\
\hline (b) & 116 & 118 & 76 & 79 & 40 & 39 \\
\hline (c) & 120 & 120 & 99 & 100 & 21 & 20 \\
\hline
\end{tabular}

Table 1. G and Y component as brightness level of images in Fig. 3 
In brief, the G component of an RGB image will be used as the luminance when estimating lighting conditions. It is the relationship between the mean and median $G$ values of an image to be used as the criterion to judge illuminating conditions. For under- and properly exposed pictures, if the difference between these two values is minor, the scene is normal lit; otherwise the scene is excessive back-lit or it pocesses a high dynamic range illumination. This relationship will be used in the AE mechanism to help control the exposure value depending on lighting conditions. In term of implementation, the hardware required to compute the mean and median value is simple and among basic blocks. Thus, this method is really effective in terms of processing time and implementation.

\subsection{Auto exposure}

The proposed AE method addresses image capturing systems that employ CMOS image sensor and that have limited capabilities. According to (Liang et al., 2007) and (Kuno et al., 1998), the relationship between the luminance value and the exposure factors can be expressed as:

$$
B l=k \times L \times G \times T \times(F / \#)^{-2}
$$

where $B l$ is the brightness level of the captured image, $k$ is a constant, $L$ is the luminance of the ambient light, $G$ is the gain of the automatic gain control, $F / \#$ is the aperture value, and $T$ is the integration time.

This basic equation is used in combination with $B l_{\text {mean }}, B l_{\text {med }}, D_{L}$, and $D_{\text {thres }}$ to enhance the proposed modified AE algorithm.

Let $B l_{n}$ and $B l_{\text {opt }}$ denote the brightness levels of the current frame and the frame taken with optimal exposure time. For a certain scene and when both frames are taken continuously within a very short time, $L$ and $G$ remain almost the same. For most cell phones and surveillance cameras employing CMOS technologies, the aperture is fixed at its maximum value, thus $F / \#$ is constant. The exposure function (1) for the current frame and the frame taken with optimal exposure time are:

$$
\begin{gathered}
B l_{n}=k \times L \times G \times T_{n} \times(F / \#)^{-2} \\
B l_{\text {opt }}=k \times L \times G \times T_{\text {opt }} \times(F / \#)^{-2}
\end{gathered}
$$

where $T_{n}$ and $T_{\text {opt }}$ are the current and optimal integration time values.

By dividing (2) by (3), the relationship between $B l_{n}$ and $B l_{\text {opt }}$ can be expressed as:

$$
\begin{gathered}
\frac{B l_{n}}{B l_{\text {opt }}}=\frac{k \times L \times G \times T_{n} \times(F / \#)^{-2}}{k \times L \times G \times T_{\text {opt }} \times(F / \#)^{-2}} \\
{\left[B l_{n} / B l_{\text {opt }}\right]=\left[T_{n} / T_{\text {opt }}\right]} \\
\log _{2} B l_{n}-\log _{2} B l_{\text {opt }}=\log _{2} T_{n}-\log _{2} T_{\text {opt }} \\
\log _{2} T_{\text {opt }}=\log _{2} T_{n}-\log _{2} B l_{n}+\log _{2} B l_{\text {opt }}
\end{gathered}
$$


The proposed algorithm uses $B l_{\text {mean }}$ to control $\mathrm{AE}$ based on the idea of mid-tone in an iterative way. The mid-tone idea assumes that the optimal exposure value should be around 128 which is the middle value of the range [0, 255]. However, unlike (Liang et al., 2007), in this paper, the optimal brightness level is not fixed. $B l_{\text {opt }}$ may be changed according to the lighting conditions. Besides, since the camera response is not totally linear, the actual values in each condition are obtained by performing a series of experiments. A lot of pictures were taken under different lighting conditions in order to obtain the most suitable optimal values of $B l_{\text {opt }}$ for normal lighting, back lighting or high contrast lighting conditions, and lighting conditions when the current picture is over exposed. These optimal values are expected to be close to the mid-tone value 128 , which means that the values of $\log _{2} B l_{\text {opt }}$ should be close to $\log _{2} 128=7$.

Let $B l_{\text {opt }}^{\text {norm }}$ denote the optimal brightness level in the case of normal-lit conditions with low exposure time, $B l_{\text {opt }}^{b k d r}$ denote the optimal value in the case of back lighting or high contrast lighting conditions with low exposure time, and let $B l_{\text {opt }}^{\text {over }}$ denote the optimal value in the case of over exposure.

In real implementation, (7) is convenient for data to be stored in look-up tables (LUT). The values of $B l_{\text {mean }}, B l_{n}$, and $T_{n}$ all reside in the range [0..255], which means that there are only 256 possible values for each of these variables. Therefore, for each variable, a LUT can be used to store the corresponding logarithm value of each possible value. Other operators in (7) are just simple additions and subtractions which consume little hardware and processing time. The mid-tone range $B l_{m t}$ is $[100,130]$. After capturing the first frame, the values of $B l_{\text {mean }}$ and $B l_{\text {med }}$ are calculated and are used to decide the value of $B l_{\text {opt }}$ as described in Fig. 4 . After this stage, the optimal exposure time is obtained using (7). Note that due to the non-linearity of sensors, this mechanism is supposed to be carried out iteratively until $B l_{\text {mean }}$ falls into $B l_{m t}$. Different appropriate values of $B l_{\text {opt }}$ help reduce the number of iterations instead of just one common $B l_{\text {opt }}$ for all lighting conditions.

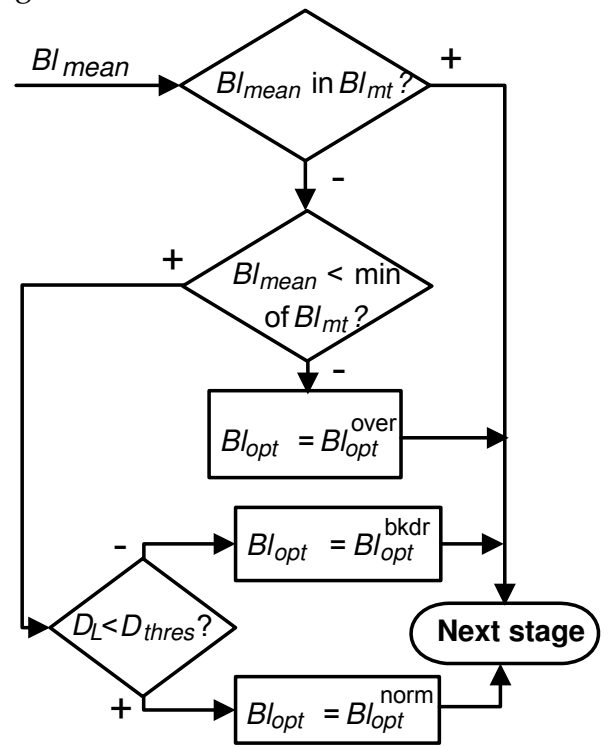

Fig. 4. Deciding value for $B l_{\text {opt }}$ 


\section{Multiple exposure}

Multiple exposure is supposed to enhance the details of an output picture by fusing multiple images of the same scene taken at different exposures. In general, multiple image fusion is really difficult to implement in terms of both complexity and speed. Image fusion also barely provides good enough quality. The main reason is image fusion involves in only luminance signal control since this mechanism is based on images of different exposure values. It is therefore hard to estimate the relationship between the luminance and the chromatic channels which is required to maintain good and real colors in the fused output image. So far it is well-known that only human eyes can do all these functions the best and in a really miracle way. Several multiple exposure algorithms have been introduced but in most cases, they tend to increase hardware cost and decrease color performance.

For low end camera systems, multiple exposure would not be a good choice due to those above reasons. The solution is to equip them with better sensors that have better dynamic range. However, this would also increase the cost. On the contrary, one more reason that limits multiple exposure performance is that existing algorithms don't consider lighting conditions when fusing images. In order to overcome those problems and make multiple exposure applicable to low end systems, this paper proposes a simple algorithm taking into account the lighting condition. The general idea of multiple exposure is described in Fig. 5 . Note that the modified $B l_{m t}$ is $[90,130]$ and is slighly different from standard $B l_{m t}$.

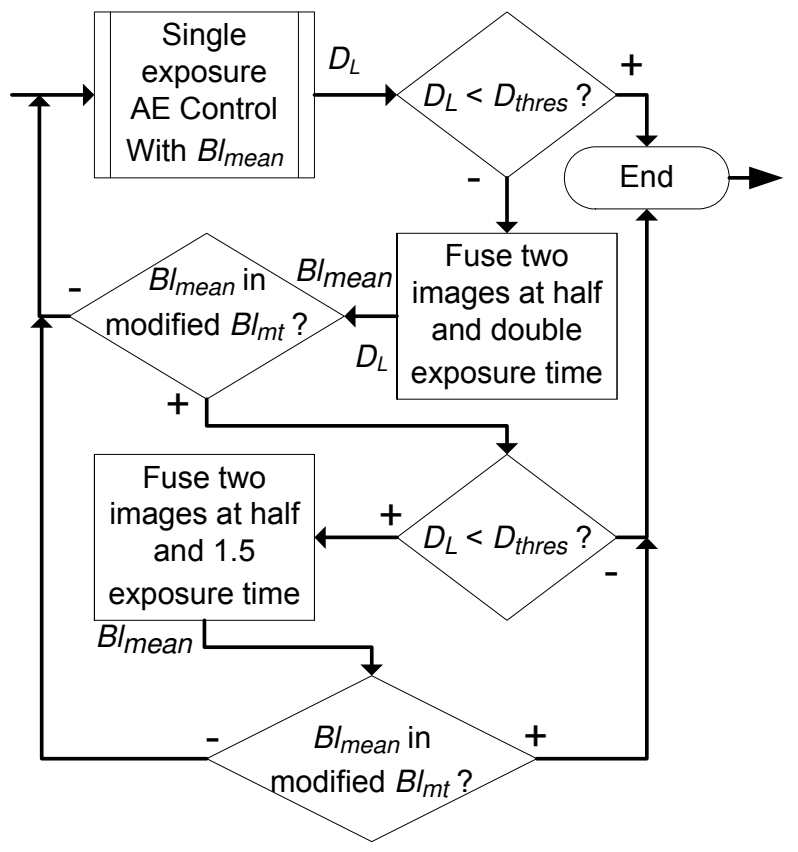

Fig. 5. Multiple exposure algorithm

The two images are simply fused together as follows:

$$
F_{X}(x, y)=\left(F_{X}^{l o}(x, y)+F_{X}^{h i}(x, y)\right) / 2
$$


where $F_{X}(x, y)$ is the color value of the pixel $(x, y), X$ is either $\mathrm{R}, \mathrm{G}$, or B component, lo is low exposure and $h i$ is high exposure. This step includes just one basic function, which is simple and easy to implement.

The multiple exposure mechanism can bring more details to dark areas and over-exposed areas. The frame taken with a lower exposure time provides details; on the other hand, the frame taken with a higher exposure time brightens the fused image.

This multiple exposure mechanism is also important to lighting condition estimation. By judging the difference values between the mean and median brightness values of an image before and after fusion, the degree of high contrast lighting can be revealed as excessive back lighting (back lighting) or just high contrast lighting.

\section{Simulations}

Simulations were carried out using a simple platform employing CMOS image sensors (CIS) with parameter values as follows:
$D_{\text {thres }}=20$
$\log _{2} B l_{\text {opt }}^{\text {norm }}=6.8$
$\log _{2} B l_{o p t}^{b k d r}=7$
$\log _{2} B l_{\text {opt }}^{\text {over }}=6.36$
$B l_{m t}=[100,130]$
Modified $B l_{m t}=[90: 130]$

Fig. 6 illustrates results of the stage of automatic exposure including the multiple exposure function since this function helps decide accurately lighting conditions. All lighting conditions were addressed during evaluation. According to Fig. 6, in the case of high dynamic range scenes, only after one image fusion can the system decide if the picture is just high contrast lit or excessive back-lit.

Simulation results show that the proposed AE algorithm can detect lighting conditions accurately and does not require much computation. Furthermore, the algorithm is independent from the position of the light source and can work well with images with or without a main object.

Because of the non-linear characteristics of CMOS sensors, sometimes it requires that the $\mathrm{AE}$ algorithm be iterated more than once since the first calculated exposure value does not return a value in the range of $B l_{\text {mean }}$ in $B l_{m t}$. Therefore, the overall $\mathrm{AE}$ mechanism may include more than one adjusting time.

Tables 2 - 4 demonstrate simulation results for all cases of lighting conditions. Both Y channel (luminance component in the $\mathrm{YCbCr}$ format) and $\mathrm{G}$ channel are observed. Simulation results show that $G$ component can be used as the luminance of an image without any significant difference. Furthermore, the lighting condition of each scene is correctly detected as its real condition. In most cases, the number of times the $\mathrm{AE}$ mechanism is iterated is less than two. This indicates that the proposed algorithm provides a high accuracy rate and fastens the overall performance.

Table 2 describes simulation results of back-lit conditions. The values of $D_{L}$ after $\mathrm{AE}$ controlling and after fusion show that fused images provide more details than un-fused ones. This ability is very useful for camera systems that employ CMOS image sensors with limited dynamic range.

In Table 3, scenes possessing high dynamic range (HDR) conditions are evaluated. After AE controlling, the multiple exposure mechanism is carried out twice. The values of $D_{L}$ also indicate that fused images provide more details than un-fused ones. 
(a) Back-lit Condition

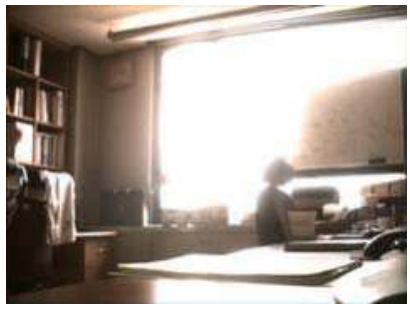

Before AE

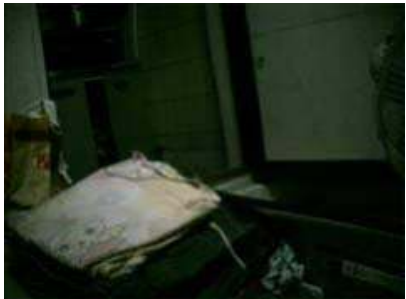

Before AE

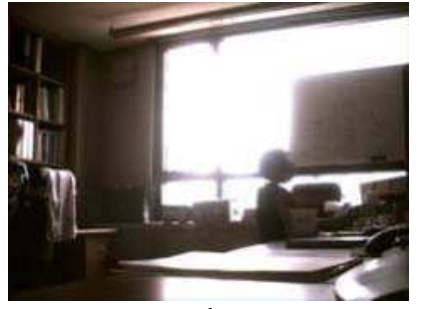

After AE

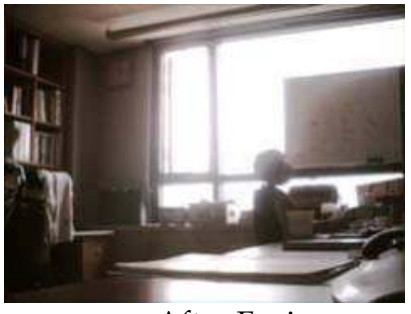

After Fusion

(b) Normal-lit Condition

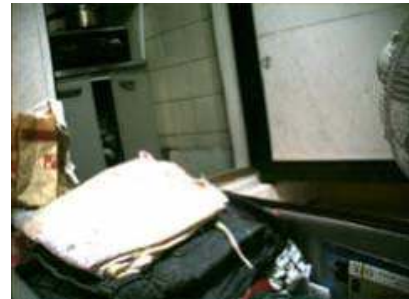

After AE

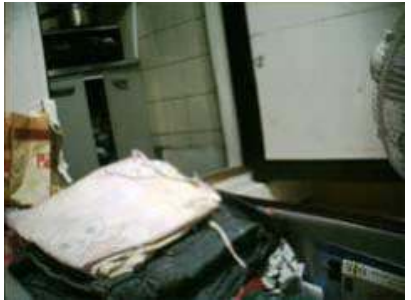

After Fusion

(c) High Contrast Lighting

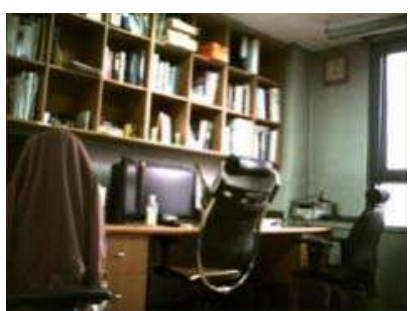

Before AE

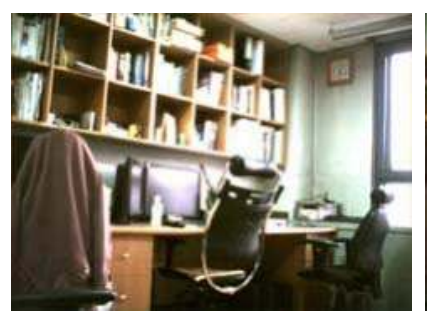

After AE

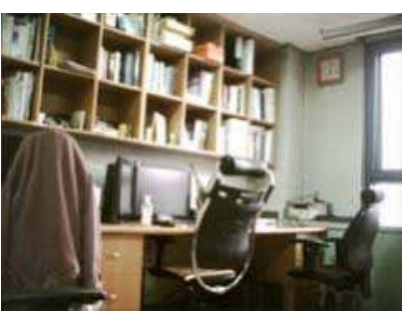

After Fusion

Fig. 6. Simulations with AE algorithm

\begin{tabular}{|c|c|c|c|c|c|c|c|c|c|}
\hline \multirow{2}{*}{ Scene } & \multirow{2}{*}{ Starting Values } & \multirow{2}{*}{ Times } & \multicolumn{3}{|c|}{ After AE } & \multicolumn{3}{|c|}{ After Fusion } \\
\cline { 6 - 7 } & & & \multirow{2}{*}{$\boldsymbol{D}_{\boldsymbol{n}}$} & $\boldsymbol{D}_{\boldsymbol{L}}$ & & \multicolumn{2}{|c|}{$\boldsymbol{B}_{\boldsymbol{n}}$} & \multirow{2}{*}{$\boldsymbol{D}_{\boldsymbol{L}}$} & \multicolumn{2}{|c|}{$\boldsymbol{B}_{\boldsymbol{n}}$} \\
\cline { 6 - 10 } & & & $\mathbf{Y}$ & $\mathbf{G}$ & & $\mathbf{Y}$ & $\mathbf{G}$ \\
\hline \hline$(1)$ & 156 & 8 & 1 & 40 & 118 & 116 & 27 & 123 & 122 \\
\hline$(2)$ & 130 & 27 & 1 & 42 & 107 & 104 & 29 & 115 & 112 \\
\hline$(3)$ & 160 & -6 & 1 & 39 & 121 & 121 & 22 & 121 & 120 \\
\hline$(4)$ & 173 & -78 & 2 & 39 & 111 & 111 & 24 & 114 & 114 \\
\hline$(5)$ & 87 & 49 & 1 & 45 & 115 & 114 & 31 & 119 & 117 \\
\hline
\end{tabular}

Table 2. Evaluation of back-lighting conditions

Table 4 describes simulation results of images taken in normal-lit conditions. The simulation also shows further values of these pictures after fusing using two images taken at half and 1.5 times the optimal exposure time. These experiment results indicate that this multiple exposure mechanism can also provide more details in output images for surveillance systems. 


\begin{tabular}{|c|c|c|c|c|c|c|c|c|c|}
\hline \multirow{3}{*}{ Scene } & \multirow{2}{*}{\multicolumn{2}{|c|}{ Starting Values }} & \multirow{3}{*}{ Times } & \multicolumn{3}{|c|}{ After AE } & \multicolumn{3}{|c|}{ After Fusion } \\
\hline & & & & \multirow{2}{*}{$D_{L}$} & \multicolumn{2}{|c|}{$B l_{n}$} & \multirow{2}{*}{$D_{L}$} & \multicolumn{2}{|c|}{$B l_{n}$} \\
\hline & $B l_{n}$ & $D_{L}$ & & & $\mathbf{Y}$ & $\mathrm{G}$ & & $\mathbf{Y}$ & $\mathrm{G}$ \\
\hline (1) & 84 & 22 & 2 & 21 & 120 & 120 & 12 & 109 & 109 \\
\hline (2) & 22 & 13 & 2 & 32 & 106 & 100 & 19 & 112 & 105 \\
\hline (3) & 77 & 29 & 2 & 25 & 115 & 114 & 13 & 107 & 106 \\
\hline (4) & 169 & -33 & 2 & 30 & 117 & 116 & 19 & 111 & 111 \\
\hline *(5) & 37 & 15 & 1 & 45 & 121 & 112 & & & \\
\hline
\end{tabular}

Table 3. Evaluation of high contrast lighting conditions

*night scene taken with the system's maximum exposure value; thus no fusion was carried out after AE.

\begin{tabular}{|c|c|c|c|c|c|c|c|c|c|}
\hline \multirow{3}{*}{ Scene } & \multirow{2}{*}{\multicolumn{2}{|c|}{ Starting Values }} & \multirow{3}{*}{ Times } & \multicolumn{3}{|c|}{ After AE } & \multicolumn{3}{|c|}{ After Fusion } \\
\hline & & & & \multirow{2}{*}{$D_{L}$} & \multicolumn{2}{|c|}{$B l_{n}$} & \multirow{2}{*}{$D_{L}$} & \multicolumn{2}{|c|}{$B l_{n}$} \\
\hline & $B l_{n}$ & $D_{L}$ & & & $\mathbf{Y}$ & G & & $\mathbf{Y}$ & G \\
\hline$\overline{(1)}$ & 79 & $\overline{-3}$ & 1 & -11 & 117 & 115 & $\overline{-14}$ & 110 & 109 \\
\hline (2) & 82 & 14 & 1 & 14 & 105 & 104 & 8 & 99 & 99 \\
\hline (3) & 8 & 3 & 3 & 15 & 109 & 106 & 8 & 99 & 98 \\
\hline (4) & 40 & 11 & 1 & 15 & 107 & 111 & 9 & 101 & 104 \\
\hline *(5) & 3 & 1 & 1 & 0 & 42 & 39 & & & \\
\hline
\end{tabular}

Table 4. Evaluation of normal lighting conditions

*night scene taken with the system's maximum exposure value.

The proposed algorithm was also applied on a hi-end digital still camera (DSC) in combination with a computer-based software for experiments. Eventhough the CCD of the DSC has a much better dynamic range than the CIS, this method still improved the ability of estimating lighting conditions as well as details of output pictures. Simulations were carried out with the same scene but under different lighting conditions to illustrate the performance of the algorithm as depicted in Fig. 7 and Fig. 8. In the case of normal-lighting (Fig. 8b), the built-in and the proposed mechanisms introduced relevant outputs in terms of exposure and details.

Evaluations were performed under the condition of no flash for better comparisons. Although the proposed algorithm can only slightly improves the performance of the DSC, it still helps estimate lighting conditions accurately.

\section{Conclusion}

A new AE algorithm with lighting condition detecting capability has been introduced. The proposed architecture mainly addresses platforms employing CMOS Image Sensor, most of which have limited capabilities. However, the new and simple method for estimating lighting conditions is also widely applicable to other hi-end platforms.

The proposed algorithm can quickly estimate an appropriate exposure value after a small number of frames. It can also improve the accuracy and enhance the details of output images, owing to the simple multiple exposure mechanism.

Using the new mechanism to detect lighting conditions, the system is flexible and can work well with most images without being affected by the positions of light sources and main objects. Since the algorithm is not computationally complicated, it can be fitted in most CMOS platforms that have limited capabilities such as cell phones and/or surveillance cameras. 


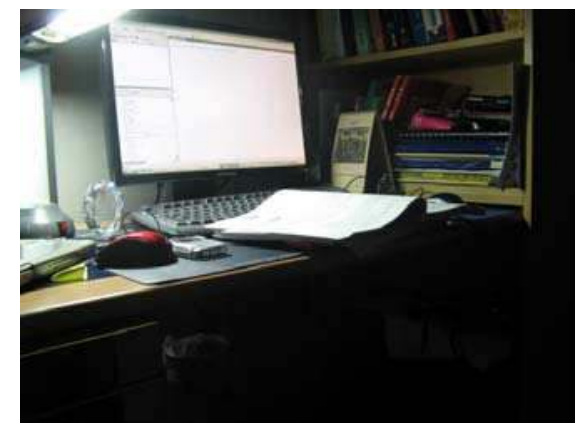

$$
\begin{array}{ll}
\text { Before } \mathrm{AE} & B l_{\text {mean }}=73 \\
& B l_{\text {med }}=23 \\
& D_{L}=50>D_{\text {thres }}
\end{array}
$$

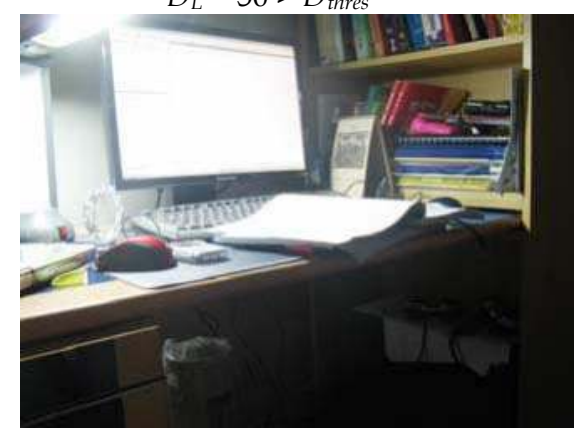

After Fusion $B l_{\text {mean }}=106$

$$
B l_{\text {med }}=69
$$

$D_{L}=37>D_{\text {thres }}$

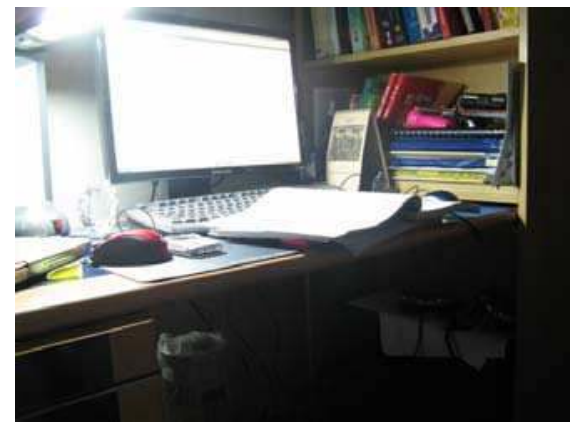

After $\mathrm{AE} \quad B l_{\text {mean }}=104$

$B l_{\text {med }}=62$

$D_{L}=42>D_{\text {thres }}$

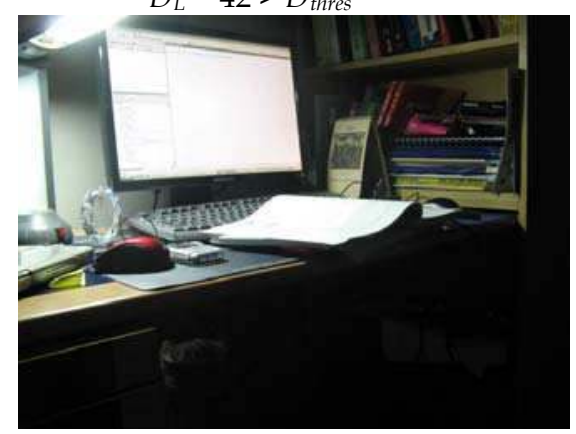

DSC Auto Mode $B l_{\text {mean }}=75$

$B l_{\text {med }}=25$

$D_{L}=50$

Fig. 7. Back-lighting/excessive lighting condition with DSC

In the future, the multiple exposure method should be further improved so that no luminance cast is introduced and the degree of lighting conditions can be more precisely estimated. Furthermore, besides AE, there are two other important ISP functions: AF, and AWB. Future research would focus on implementing these two functions such that the relationship between the mean and the median values of each color channel can be further exploited, thus the resource and the result of $\mathrm{AE}$ stage can be re-used to reduce the computing time and the hardware required.

\section{References}

Kao, W. C.; Hsu, C. C.; Kao, C. C. \& Chen, S. H. (2006). Adaptive exposure control and realtime image fusion for surveillance systems. Proceedings of IEEE Int. Symposium on Circuits and Systems, vol. 1-11, pp. 935-938, Kos, Greece, May 2006.

Kuno, T.; Sugiura, H. \& Atoka, M. (1998). A new automatic exposure system for digital still cameras. IEEE Trans. Consum. Electron., vol. 44, pp. 192-199, Feb. 1998.

Lee, J. S.; Jung, Y. Y.; Kim, B. S. \& Ko, S. J. (2001). An advanced video camera system with robust AF, AE, and AWB control. IEEE Trans. Consum. Electron., vol. 47, pp. 694699, Aug. 2001. 
(a) High Contrast Lighting

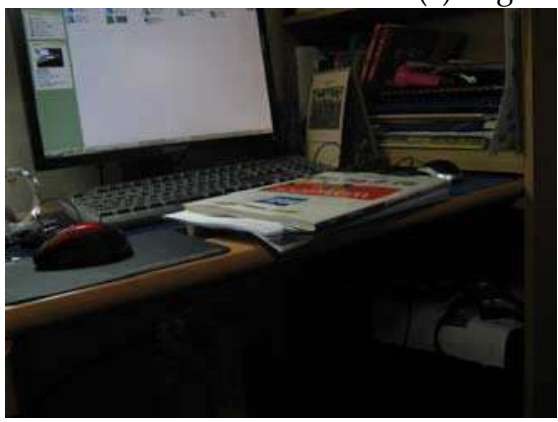

Before AE

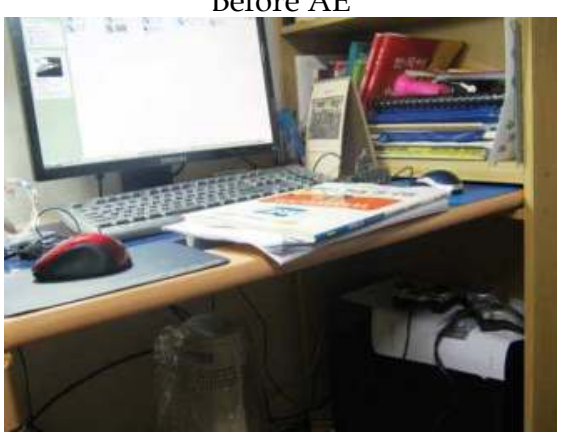

After Fusion

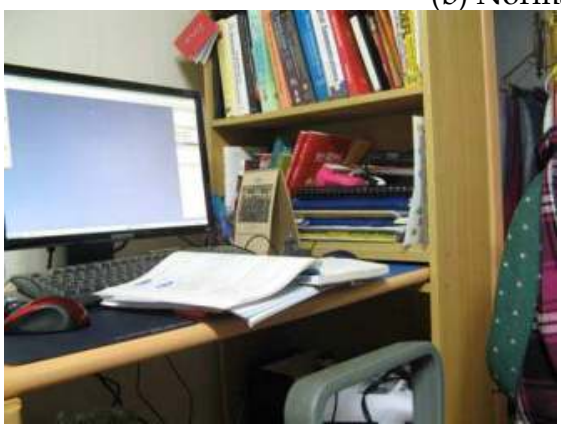

Before \& After AE
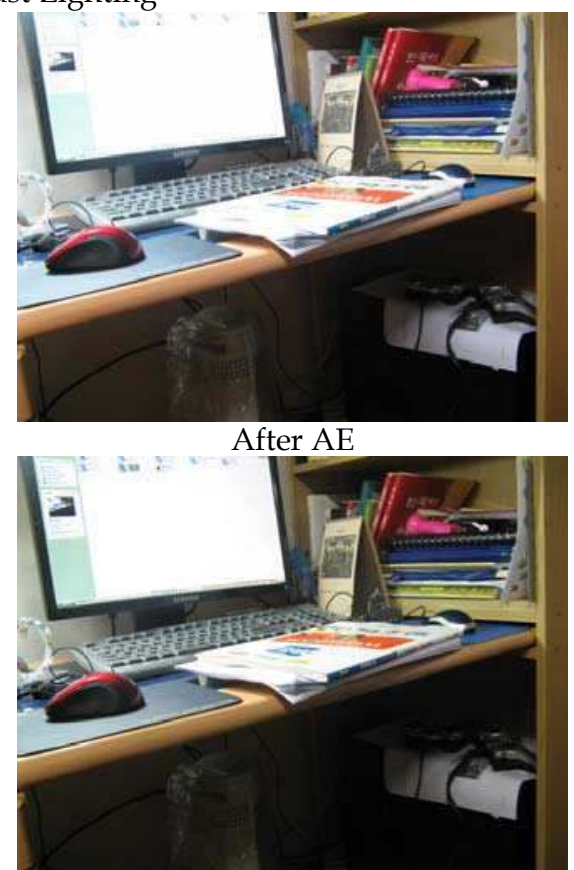

DSC Auto Mode

(b) Normal-lit Condition

Fig. 8. High dynamic range and normal-lighting conditions with DSC

Liang, J. Y.; Qin, Y. J. \& Hong, J. L (2007). An auto-exposure algorithm for detecting high contrast lighting conditions. Proceedings of the 7th Int. Conf. on ASIC, vols. 1 and 2, pp. 725-728, Guilin, Peoples R. China, Oct. 2007.

Murakami, M. \& Honda, N. (1996). An exposure control system of video cameras based on fuzzy logic using color information. Proceedings of 5th IEEE Int. Conf. on Fuzzy Systems, vols 1-3, pp. 2181-2187, Los Angeles, Sep. 1996.

Shimizu, S.; Kondo, T.; Kohashi, T.; Tsuruta, M. \& Komuro, T. (1992). A new algorithm for exposure control based on fuzzy logic for video cameras. IEEE Trans. Consum. Electron., vol. 38, pp. 617-623, Aug. 1992. 


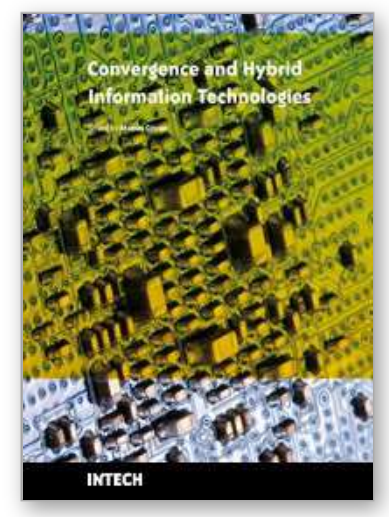

\author{
Convergence and Hybrid Information Technologies \\ Edited by Marius Crisan
}

ISBN 978-953-307-068-1

Hard cover, 426 pages

Publisher InTech

Published online 01, March, 2010

Published in print edition March, 2010

Starting a journey on the new path of converging information technologies is the aim of the present book. Extended on 27 chapters, the book provides the reader with some leading-edge research results regarding algorithms and information models, software frameworks, multimedia, information security, communication networks, and applications. Information technologies are only at the dawn of a massive transformation and adaptation to the complex demands of the new upcoming information society. It is not possible to achieve a thorough view of the field in one book. Nonetheless, the editor hopes that the book can at least offer the first step into the convergence domain of information technologies, and the reader will find it instructive and stimulating.

\title{
How to reference
}

In order to correctly reference this scholarly work, feel free to copy and paste the following:

Quoc Kien Vuong, Se-Hwan Yun and Suki Kim (2010). A New Auto Exposure System to Detect High Dynamic Range Conditions Using CMOS Technology, Convergence and Hybrid Information Technologies, Marius Crisan (Ed.), ISBN: 978-953-307-068-1, InTech, Available from:

http://www.intechopen.com/books/convergence-and-hybrid-information-technologies/a-new-auto-exposuresystem-to-detect-high-dynamic-range-conditions-using-cmos-technology

\section{INTECH}

open science | open minds

\author{
InTech Europe \\ University Campus STeP Ri \\ Slavka Krautzeka 83/A \\ 51000 Rijeka, Croatia \\ Phone: +385 (51) 770447 \\ Fax: +385 (51) 686166 \\ www.intechopen.com
}

\author{
InTech China \\ Unit 405, Office Block, Hotel Equatorial Shanghai \\ No.65, Yan An Road (West), Shanghai, 200040, China \\ 中国上海市延安西路65号上海国际贵都大饭店办公楼405单元 \\ Phone: +86-21-62489820 \\ Fax: +86-21-62489821
}


(C) 2010 The Author(s). Licensee IntechOpen. This chapter is distributed under the terms of the Creative Commons Attribution-NonCommercialShareAlike-3.0 License, which permits use, distribution and reproduction for non-commercial purposes, provided the original is properly cited and derivative works building on this content are distributed under the same license. 